\title{
TERAPÊUTICA MEDICAMENTOSA: CONHECIMENTO E DIFICULDADES DE FAMILIARES DE PESSOAS IDOSAS COM TRANSTORNO AFETIVO BIPOLAR ${ }^{1}$
}

\author{
Maristela Monteschi², Kelly Graziani Giacchero Vedana ${ }^{3}$, Adriana Inocenti Miasso ${ }^{4}$
}

\footnotetext{
${ }^{1}$ Projeto financiado pelo Programa Institucional de Bolsa de Iniciação Científica - PIC/RUSP e pelo CNPq.

${ }^{2}$ Mestranda do Programa de Pós-Graduação em Enfermagem Psiquiátrica da Escola de Enfermagem de Ribeirão Preto (EERP) da Universidade de São Paulo (USP). São Paulo, Brasil. E-mail: maristelamonteschi@yahoo.com.br

${ }^{3}$ Doutoranda do Programa de Pós-Graduação em Enfermagem Psiquiátrica da EERP/USP. Enfermeira Especialista em Laboratório do Departamento de Enfermagem Psiquiátrica e Ciências Humanas da EERP/USP. São Paulo, Brasil. E-mail: kellygiacchero@eerp.usp.br

${ }^{4}$ Doutora em Enfermagem. Professora Doutora do Departamento de Enfermagem Psiquiátrica e Ciências Humanas da EERP/ USP. São Paulo, Brasil. E-mail: amiasso@eerp.usp.br
}

\begin{abstract}
RESUMO: Este estudo descritivo quali-quantitativo teve como objetivo verificar o grau de conhecimento e dificuldades do familiar do idoso com Transtorno Afetivo Bipolar referentes ao tratamento medicamentoso prescrito para o paciente. Participaram do estudo 17 familiares de idosos com Transtorno Afetivo Bipolar atendidos em um Núcleo de Saúde Mental. Para coleta de dados empregou-se a entrevista semiestruturada e aplicação de escala. Identificou-se baixo nível de conhecimento dos familiares, especialmente sobre doses e frequência dos medicamentos prescritos. Dentre as dificuldades relacionadas ao tratamento medicamentoso dos pacientes, relatadas pelos familiares, destacaram-se a ambiguidade em relação à avaliação dos benefícios do medicamento, não aderência dos pacientes ao medicamento, sobrecarga relacionada aos cuidados referentes aos medicamentos, tratamento inadequado, preocupação com o acesso ao medicamento e utilização de bebida alcoólica pelo paciente. É imperativa a necessidade de ações educativas junto à família e sua inclusão na assistência oferecida ao paciente, como colaboradora e alvo das intervenções.
\end{abstract}

DESCRITORES: Transtorno bipolar. Adesão à medicação. Idoso. Família. Enfermagem.

\section{MEDICATION THERAPY:KNOWLEDGE AND DIFFICULTIES OF RELATIVES OF ELDERLY PEOPLE WITH BIPOLAR AFFECTIVE DISORDER}

\begin{abstract}
This study descriptive qualitative and quantitative aimed to verify the level of knowledge and difficulties of relatives of elderly people with Bipolar Affective Disorder regarding the medication treatment prescribed to patients. The participants were 17 relatives of elderly patients with Bipolar Affective Disorder who receive care in a Mental Health Service. Semi-structured interview and a scale were used for data collection. Low level of knowledge from relatives was verified, especially concerning the doses and frequency of the prescribed medications. Among the difficulties related to patients' medication treatment reported by the relatives, the following were pointed out: ambiguity regarding the evaluation of the medication benefits, non-adherence of patients to the medication, overload related to care concerning medications, inappropriate treatment, preoccupation with the access to medication and use of alcoholic drinks by patients. It is imperative to offer educational actions to the families, as collaborators and target of the interventions, as well as inserting them in the care provided to patients.
\end{abstract}

DESCRIPTORS: Bipolar disorder. Medication adherence. Aged. Family. Nursing.

\section{TERAPÉUTICA MEDICAMENTOSA: CONOCIMIENTO Y DIFICULTADES DE FAMILIARES DE PERSONAS ANCIANAS CON TRASTORNO AFECTIVO BIPOLAR}

\begin{abstract}
RESUMEN: Este estudio cualitativo y cuantitativo tuvo como objetivo determinar el grado de conocimiento y las dificultades de los familiares de ancianos con Trastorno Afectivo Bipolar relacionados al tratamiento farmacológico prescrito al paciente. Participaron del estudio 17 familiares de ancianos con Trastorno Afectivo Bipolar que reciben atención en un Servicio de Salud Mental. Para la recolección de datos se utilizó la entrevista semi-estructurada y la aplicación de escala. Se identificó bajo nivel de conocimiento de los familiares, especialmente acerca de las dosis y frecuencia de los medicamentos prescritos. Entre las dificultades relacionadas al tratamiento farmacológico, relatadas por los familiares, se destacan la ambigüedad en relación a la evaluación de los beneficios del medicamento, no adherencia de pacientes al medicamento, sobrecarga relacionada con la atención referente a los medicamentos, tratamiento inadecuado, preocupación con el acceso al medicamento y utilización de bebida alcohólica por los pacientes. Son necesarias acciones educativas junto a las familias, como colaboradoras y blanco de las intervenciones, y su inclusión en la atención ofrecida al paciente

DESCRIPTORES: Transtorno bipolar. Cumplimiento de la medicación. Anciano. Familia. Enfermería.
\end{abstract}




\section{INTRODUÇÃO}

O Transtorno Afetivo Bipolar (TAB) na terceira idade é um sério problema de saúde pública e representa um enorme desafio para o tratamento clínico. ${ }^{1-2}$ Esse transtorno usualmente acomete indivíduos jovens, portanto a maior parte dos casos de TAB em idosos representa uma fase de um transtorno de humor que se iniciou há vários na vida do indivíduo. Entretanto, o TAB também pode surgir na idade avançada. ${ }^{3-4}$

Existe uma carência de estudos epidemiológicos sobre o TAB geriátrico. ${ }^{5}$ Nos Estados Unidos, as taxas de prevalência de TAB em idosos variam entre $0,1 \%$ e $0,4 \%$. No entanto, este transtorno atinge cerca de $10 \%$ a $25 \%$ de todos os pacientes idosos com transtornos de humor e é responsável por 5\% das internações de idosos por causas psiquiátricas no país. ${ }^{1}$ No Brasil, não há uma precisão sobre a incidência e prevalência de TAB na terceira idade. Entretanto, o diagnóstico de TAB em idosos destaca-se como causa de internações psiquiátricas neste grupo. ${ }^{5}$

É fato que idosos portadores de TAB frequentemente têm diferentes necessidades de tratamento em comparação com indivíduos jovens. Estas são decorrentes de fatores típicos da terceira idade: comorbidades físicas, isolamento social, perdas cognitivas, polifarmácia, variações relacionadas à idade na resposta à terapia, entre outras. ${ }^{1}$ Além disso, idosos com TAB podem apresentar declínio cognitivo mais rápido do que o esperado para pessoas na mesma faixa etária e com a mesma escolaridade. Esta alteração pode propiciar maior dependência dos idosos em relação aos cuidados prestados por familiares ou outros cuidadores. ${ }^{6}$

Os estudos sobre TAB em idosos são limitados. Infelizmente, o manejo do transtorno bipolar geriátrico tem sido relativamente pouco estudado em comparação com o tratamento da população jovem..$^{1-2,7}$ Uma revisão da literatura sobre a temática, trabalhando em diferentes bases de dados, identificou apenas 61 artigos que abordavam o assunto. Os autores apontam a necessidade de investigações nessa clientela visando melhorias na qualidade da assistência direcionada à mesma, ${ }^{7} \mathrm{o}$ que evidencia a relevância da presente pesquisa.

Atualmente as alternativas disponíveis para o tratamento do $\mathrm{TAB}$ no idoso usualmente incluem estabilizadores do humor (lítio, anticonvulsivantes) e psicoterapia. Também podem ser utilizados, em alguns casos, os antipsicóticos, ansiolíticos, antidepressivos e a eletroconvulsoterapia. ${ }^{1}$
Como o TAB é crônico, a aderência ao tratamento medicamentoso é fundamental para o sucesso do mesmo. Porém, existe um grande número de barreiras a serem transpostas pela pessoa com TAB diante da necessidade de uso contínuo de medicamentos, resultando, frequentemente, na não adesão aos mesmos. ${ }^{8}$ Em especial para o idoso com TAB esta é uma questão crítica, tendo em vista que, com frequência, se encontra em uso de outros medicamentos ${ }^{7}$ além daqueles prescritos para o transtorno mental. Destaca-se que a não adesão ao medicamento pode aumentar a recorrência de mania, a frequência de episódios depressivos, hospitalizações e suicídios, ${ }^{7,9}$ gerando sofrimento tanto para os pacientes como para seus familiares.

Nesse contexto, os familiares que buscam suporte nos serviços de saúde mental apresentam demandas das mais variadas ordens, dentre elas, a dificuldade para lidarem com as situações de crise vividas, com os conflitos familiares emergentes, com a culpa e o pessimismo, pelas dificuldades materiais da vida cotidiana, pela complexidade do relacionamento com o paciente, sua expectativa frustrada de cura, bem como pelo desconhecimento da doença propriamente dita e do tratamento prescrito. ${ }^{10}$

Faz-se, assim, necessário identificar possibilidades de intervenção e de ajuda junto à família, bem como efetivá-la como colaboradora e alvo do cuidado. Para tanto, ela precisa conhecer os vários aspectos relacionados ao transtorno e à terapêutica medicamentosa do paciente, como sinais e sintomas indicativos de recorrência e recaída, nome dos medicamentos prescritos, dose, efeitos terapêuticos e adversos dos mesmos, dentre outros. ${ }^{11}$ Alguns trabalhos têm evidenciado que a inclusão de familiares no tratamento de pessoas com $\mathrm{TAB}$ tem efeito positivo no curso do tratamento, havendo, assim, maior entendimento e aceitação do transtorno. ${ }^{12}$

Desse modo, a proposta de estudar o conhecimento e as dificuldades de familiares de idosos com $\mathrm{TAB}$ a respeito do tratamento medicamentoso vem ao encontro da carência de estudos relacionados à temática e da necessidade de se otimizar o tratamento dispensado a essa clientela.

\section{OBJETIVOS}

Este estudo teve como objetivo verificar o grau de conhecimento e as dificuldades do familiar do idoso com TAB referentes ao tratamento medicamentoso prescrito para o paciente. 


\section{METODOLOGIA}

Trata-se de um estudo descritivo, com abordagem quali-quantitativa. Destaca-se que os dados quantitativos referentes ao conhecimento do familiar acerca da terapêutica medicamentosa prescrita para o paciente foram utilizados para complementar aqueles de natureza qualitativa.

A pesquisa foi realizada no domicílio de familiares de pessoas idosas com TAB atendidas em um Núcleo de Saúde Mental (NSM) pertencente ao Sistema Único de Saúde, localizado em um município do interior paulista. O projeto foi desenvolvido após sua aprovação pelo Comitê de Ética em Pesquisa da instituição (Protocolo n ${ }^{\circ} 269$ / CEP-CSE-FMRP-USP) e foi solicitado aos participantes que assinassem o termo de consentimento pós-esclarecido.

Para seleção dos sujeitos, inicialmente foi realizado um levantamento de todos os pacientes com 60 anos ou mais, diagnosticados com TAB, com prescrição de uso contínuo de medicamentos para tratamento do referido transtorno, e que tiveram consulta agendada com os médicos do NSM durante o período de três meses após início da coleta de dados. Em seguida, cada paciente (que atendesse aos critérios descritos previamente) indicou o familiar mais envolvido ou responsável pelo seu tratamento para participar do estudo. Foram adotados, ainda, como critérios de inclusão dos familiares: ter idade igual ou superior a 18 anos e ser capaz de se comunicar verbalmente.

A coleta de dados foi realizada pelas autoras do estudo nos meses de março, abril e maio de 2009. Para coleta dos dados foi empregada a entrevista semiestruturada gravada, norteada por um roteiro elaborado pelas autoras do estudo contendo questões relativas aos dados sociodemográficos dos pacientes e familiares e uma escala que permite avaliar o conhecimento do familiar sobre a terapêutica medicamentosa instituída para o paciente. ${ }^{13-14} \mathrm{O}$ roteiro conteve ainda, questões abertas para explorar as dificuldades dos familiares relacionadas ao seguimento da farmacoterapia pelo paciente.

Para avaliação do grau de conhecimento do familiar em relação aos medicamentos utilizados, adotou-se uma escala já empregada em estudos anteriores. ${ }^{13-14}$ Tal escala permite avaliar o grau de conhecimento do familiar em relação à terapêutica medicamentosa considerando cada um dos seguintes aspectos: nome, dose e frequência de utilização. Cada item é avaliado separadamente, recebendo uma pontuação. O referido instrumento ${ }^{13}$ foi criado com o auxílio de um conjunto de es- pecialistas, embora tenha sido denominado "escala de conhecimento", apenas indica como traduzir a quantidade de informações que o paciente possui (em números) para porcentagens, a fim de facilitar a apresentação dos resultados.

A escala pressupõe que o grau de conhecimento de um sujeito sobre cada aspecto relacionado aos medicamentos pode ser pontuado de 0 a $100 \%$ e classificado em intervalos regulares que representam as seguintes classes: sem conhecimento $(0 \%)$; muito pouco conhecimento $(0 \%-25 \%)$; pouco conhecimento $(25 \%-50 \%)$; conhecimento regular $(50 \%-75 \%)$ e bom conhecimento $(75 \%-100 \%)$.

A resposta a cada pergunta foi classificada como certa ou errada, considerando-se os itens avaliados para cada um dos medicamentos prescritos para o paciente. A resposta "não sei" foi classificada como errada. Desse modo, ao serem prescritos 10 medicamentos para um paciente, se o familiar sabe informar corretamente o nome de cinco deles, o grau de conhecimento do familiar é considerado como sendo de $50 \%$ em relação aos nomes dos medicamentos e tal familiar é incluído na categoria "pouco conhecimento" que corresponde ao intervalo de pontuação $25 \%-50 \%$. Procedeu-se dessa forma para as demais variáveis relacionadas ao medicamento.

As informações relativas à caracterização dos participantes do estudo e ao conhecimento dos mesmos sobre a terapêutica medicamentosa foram analisadas utilizando-se estatística descritiva. Os dados foram digitados em uma base de dados estruturada no formato de planilha, no programa Excel, e posteriormente transportados para o programa Statistical Package for the Social Science (SPSS, versão 11.5).

Para a análise dos dados sobre as dificuldades dos familiares referentes ao seguimento da terapia medicamentosa pelo paciente, foi utilizada a abordagem qualitativa seguindo os seguintes passos: ${ }^{15}$ ordenação dos dados coletados, classificação dos dados (desenvolvimento das categorias empíricas centrais) e análise final (articulação dos dados empíricos com a literatura).

Os resultados serão apresentados em três tópicos distintos: a caracterização dos participantes do estudo, o grau de conhecimento dos familiares sobre os medicamentos prescritos para os idosos com TAB e as dificuldades de familiares de pessoas idosas com TAB relacionadas à terapêutica medicamentosa. É válido ressaltar que, para garantir o anonimato dos familiares participantes, estes 
foram identificados com a letra F acrescida do número que corresponde à ordem em que ocorreram as entrevistas.

\section{RESULTADOS}

\section{Categorização dos sujeitos do estudo}

Tiveram consultas médicas agendadas no período do estudo e preencheram os critérios de inclusão, 17 pessoas idosas com TAB. Dessa forma, foram incluídos na pesquisa os 17 familiares mencionados como as pessoas mais envolvidas no tratamento medicamentoso desses pacientes. No que se refere às características sociodemográficas, constatou-se que a maioria das pessoas responsáveis pela provisão de cuidados aos idosos portadores de TAB era do sexo feminino $(76,47 \%)$, casada $(76,47 \%)$, não havia completado o ensino fundamental $(58,80 \%)$ e referiu que a renda per capita da família era igual ou menor que um salário mínimo (58,80\%). Apenas três entrevistados $(17,64 \%)$ possuíam vínculo empregatício.

\section{O grau de conhecimento sobre o medica- mento}

No presente estudo, foi avaliado o conhecimento dos familiares de pessoas idosas com TAB em relação aos medicamentos prescritos pelo psiquiatra, comparando-se a última prescrição médica disponível no prontuário do paciente com os medicamentos referidos pelo familiar. Foi analisado o grau de conhecimento referente ao nome, dose e frequência dos medicamentos, conforme mostra o Gráfico 1.

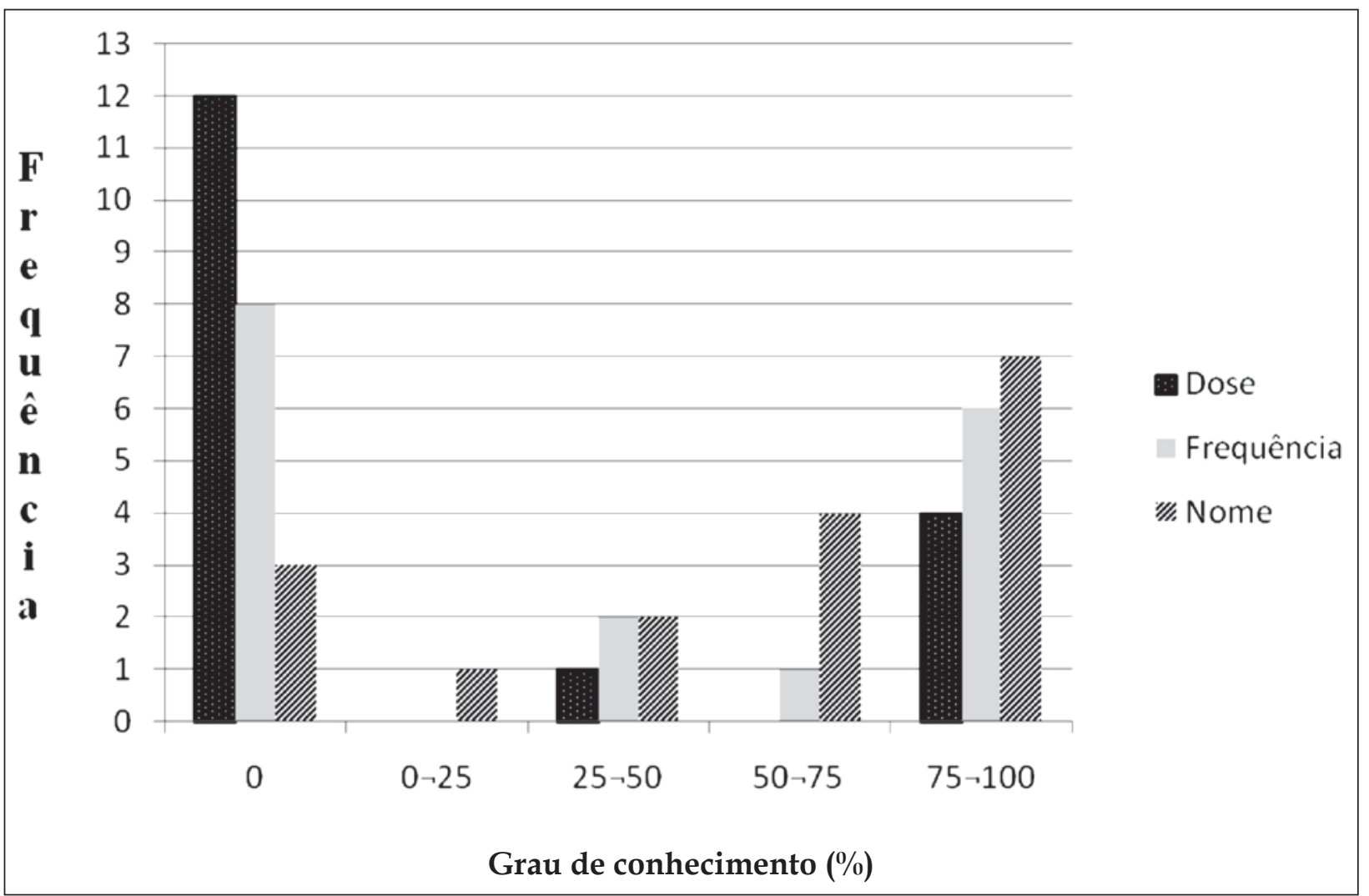

Gráfico 1 - Distribuição dos sujeitos do estudo de acordo com o grau de conhecimento da dose, frequência e nome do medicamento

Para a avaliação do conhecimento em relação ao nome do medicamento, foram consideradas as respostas que identificassem tanto o nome genérico como o nome comercial do mesmo. Dessa forma, no total de 17 entrevistados, encontrou-se que sete $(41,18 \%)$ apresentaram bom conhecimento sobre o nome do medicamento utilizado pelo paciente.
Destaca-se que três familiares $(17,65 \%)$ não tinham qualquer conhecimento a esse respeito.

Observou-se que, entre os 17 sujeitos da pesquisa, a maioria $(70,59 \%)$ não conhecia a dose correta da medicação prescrita para o paciente. Um (5,88\%) familiar possuía pouco conhecimento e apenas quatro $(23,53 \%)$ entrevistados foram 
avaliados como tendo bom conhecimento sobre a dose da medicação.

Muitos entrevistados $(47,06 \%)$ não conheciam a frequência de utilização dos medicamentos prescritos para o idoso com $\mathrm{TAB}$ e apenas seis $(35,29 \%)$ familiares apresentaram bom conhecimento a esse respeito.

\section{As dificuldades de familiares de pessoas idosas com $\mathrm{TAB}$ relacionadas à terapêutica medicamentosa}

Os familiares descreveram várias dificuldades cotidianas em relação ao seguimento da terapêutica medicamentosa pelos idosos com TAB. Na análise qualitativa das falas dos sujeitos referentes a essas dificuldades, emergiram categorias que serão apresentadas em subtópicos, na sequência.

\section{Avaliando efeitos do tratamento medica- mentoso}

Esta categoria descreve o dilema no qual muitos familiares se encontravam ao avaliar os aspectos positivos e negativos decorrentes do uso contínuo dos medicamentos. Seus depoimentos revelam um julgamento ambíguo em relação aos efeitos do medicamento utilizado pelo paciente. Embora a maioria dos familiares valorizasse os benefícios proporcionados pela medicação, muitos identificaram efeitos colaterais e ineficácia eu/ou prejuízo da farmacoterapia, expressando preocupação a esse respeito.

Ela não tem condições de ficar sem tomar o medicamento [...]. Ela não tem mais estrutura para ficar sem medicamento [...] mas o remédio que ela tava tomando estava fazendo mal [...] (F4).

Melhorou bastante, melhorou... parou com as crises nervosas, aquelas coisas [...]. Ela queixa de dor no estômago, fala que queima, eu falo para ela tomar tudo com leite [...]. Os remédios são forte não é? (F6).

Foi o $\mathrm{Dr} A$. que receitou o remédio pra ele, mas deu tremores, muitos tremores [...]. Ai, daí um tempo deu um nó nas tripa, que ele não conseguia fazer cocô, porque ele tomava esses remédios e deu isso (F15).

Alguns familiares apontaram como negativa a possibilidade da ocorrência de crises e recaídas, mesmo com a utilização adequada dos medicamentos prescritos.

Antes, quando ele tinha crise, ele tomava medicação, tomava, mas mesmo assim dava crise. Só quando dava a crise, né... quando eu pensava que ele estava melhorando, de repente vinha a crise (F2).
Porque tem altos e baixos, um dia tá bem, outro ela não está (F8).

\section{Deparando-se com a não aderência do paciente e sobrecarga nos cuidados relacionados aos medicamentos}

Verificou-se que muitos familiares relataram o problema da não aderência como uma dificuldade enfrentada em relação à terapêutica medicamentosa, especialmente quando o idoso com TAB era o responsável pela administração de seu medicamento.

Teve um período que tava meio que por conta dele, mas aí a gente começou a perceber que teve dia que ele tomava certinho e teve dia que não [...] (F13).

Ela falava assim: "Ai eu já tô boa." - e jogava fora a medicação. Aí era onde dava crise, e ela entrava em crise e ficava um ano em crise (F17).

Foi possível perceber que algumas vezes o comportamento de não aderência ao tratamento medicamentoso foi intencional, ou seja, o paciente não aderia à farmacoterapia por vontade própria. Diante desse problema, alguns familiares precisavam monitorar os pacientes na administração dos medicamentos.

Ela não gosta de tomar remédio, é isso [...]. Agora ela toma, mas eu não sei se eu tivesse que deixar ela tomar todo dia, se ela tomaria [...]. Eu não sei se na minha falta ela vai tomar o remédio direitinho (F10).

Tem vez que ela não quer saber de tomar remédio... Você tem que ficar de olho e ver se ela tomou mesmo, porque já teve vezes dela esconder debaixo da lingua e você achar que ela tomou [...]. Eu tive que pegar medicação, desmanchar no suco porque eu vi que ela não tava tomando (F16).

Entretanto, nem sempre o familiar tinha êxito na monitoração da aderência do paciente. No exemplo a seguir, a familiar tem limitações para intervir na utilização do medicamento por medo da agressividade do paciente, sendo coagida a mentir ou não acompanhá-lo na consulta médica.

Ele não está tomando... ele não toma... Não adianta eu falar, porque se eu vou falar, a gente acaba em discussão e tenho medo até de ele me agredir... Ele disse: "É muito simples eu te oriento e você fala o que eu te orientar." Eu disse: Você que sabe, eu continuo com a mentira sua [de que toma os medicamentos]. Eu não sei se ele vai deixar eu vir com ele [na consulta médica] (F5).

Identificou-se, em outros casos, que a não aderência era decorrente de uma dificuldade do 
paciente em lembrar-se de tomar o medicamento. Também nesses casos, o familiar assumiu a função de auxiliar na sua administração.

Na época que ela tava ruim, eu que dava, senão ela esquecia (F3).

Contudo, foi também expressa a dificuldade do familiar para lembrar-se de todos os medicamentos utilizados pelo paciente, especialmente quando havia prescrição de uma grande quantidade de medicamentos.

Eu anoto, uso um papel, porque eu sabia que não ia lembrar de todos (F4).

Nos depoimentos dos familiares, a não aderência é apontada tanto como "fator causal" para recaídas e crises.

Ele fez anos de tratamento, depois ele abandou o tratamento. Ai, depois de um tempo, deu a crise (F2).

Ela esquecia [de tomar os medicamentos]. Então, acho que aí ela piorava, e a gente só ia perceber quando já tava ficando ruim (F1).

Quando ela estava em crise, ela dava trabalho para tomar as medicações (F14).

Os depoimentos dos familiares refletem a sobrecarga por eles vivenciada devido à responsabilidade assumida nos cuidados relacionados aos medicamentos de idosos com TAB.

É difícil, eu vou te falar. Não é fácil não, mas eu faço a minha parte. O que está no meu alcance eu faço para ele. Ele não é criança pra eu carregar no colo... porque, uma criança, você fala: Vem! E leva na marra, mas adulto não tem como. To cansada (F5). $[\ldots](\mathrm{F} 1)$.

Ter uma pessoa doente em casa é meio complicado

\section{Julgando que o paciente recebeu tratamento inadequado}

Embora muitos familiares relatassem ocasiões em que a alteração na medicação foi necessária (para aliviar os sintomas do transtorno ou atenuar os efeitos colaterais da medicação), percebeu-se que, algumas vezes, a mudança na prescrição dos fármacos foi acompanhada de consequências indesejadas. Alguns relatos assinalam a redução da eficácia do medicamento utilizado, decorrente da diminuição da dosagem do mesmo.

De diminuir o remédio, aí ela ficava atacada, não parava de falar (F8).

Aí eu senti que ele tava bem, mas como o doutor suspendeu o remédio de uma vez e nem diminuiu.... aí veio uma crise, mas veio pior... ele estava em surto (F15).
Houve familiares que julgavam que o paciente havia recebido tratamento inadequado. Observou-se a ocorrência de tratamento que foi inicialmente oferecido ao paciente sem a prescrição de medicamentos para uso contínuo.

Ele [médico] dava Haldol, dava Akineton, mas não dava quantidade para continuar. Quando eu passei com o Dr J. ele receitou o Carbolitium (F3).

Ela ia no médico, ele dava calmante para ela. Saía de lá, tinha melhorado e ia para casa, e acontecia de novo [...]. E foi isso várias vezes [...]. Ela ia no médico, ele resolvia assim o momento, depois ia para casa e outra vez! (F11).

Também foi avaliado negativamente o tratamento oferecido quando o diagnóstico de TAB ainda não estava estabelecido.

Antes de ele ser diagnosticado bipolar, ele fez tratamento com antidepressivos. Aí ele achava que ele ficava muito debilitado com os remédios (F13).

Os depoimentos dos familiares, a seguir, revelaram a suspeita de que a prescrição fornecida pelo médico continha erro. Em um dos casos, o erro é atribuído ao descuido de um profissional.

Aí o médico disse: 'Nossa! Tá assim... Ela tem que voltar no psiquiatra por causa da dosagem. Essa dosagem não está boa'. Aí, eu voltei com ela no médico e, graças a Deus, ela melhorou (F10).

O farmacêutico falou que a receita tava errada, que não podia ser aqueles remédios para ele [paciente]. Aí, o doutor $M$. tava de férias ainda e quem tinha receitado aqueles remédios era o Dr C. Ele pegou a pasta do meu marido e nem leu, nem viu o que ele tomava antes e já foi receitando. Esse médico tava errado. Onde se viu? Dá uns remédios desse, que ele ficava pior (F15).

Neste estudo, um dos sujeitos entrevistados relatou que o paciente foi orientado pelo psiquiatra a procurar um centro espírita.

Aí ele teve no Dr D., explicou para o Dr D., e ele [médico] falou para ele [paciente] procurar o centro espírita: 'O problema dele é para o centro espírita.'. E ele [paciente] pediu para uma amiga achar um centro espírita para ele (F5).

\section{Preocupando-se com o acesso ao medica- mento}

Verificou-se que nem sempre o medicamento prescrito encontrava-se disponível na rede pública. Dessa forma, a disponibilidade do medicamento, por vezes, é um fator preocupante para os familiares dos idosos com TAB. 
Às vezes tem sim que conseguir algum remédio que tá faltando, esse Rivotril às vezes tá faltando (F3).

A dificuldade de acesso ao medicamento tem, ainda, um fator agravante que é a condição da família para comprá-lo, caso o mesmo venha a faltar.

O meu salário dá, tipo, para comprar arroz, feijão, essas coisas. Do meu dinheiro não sobra nada, às vezes sobra um 100 cruzeiro, 100 real, para pagar água, para pagar outras coisas, não dá para comprar o Carbolitium (F3).

Devez em quando tem que comprar [o dinheiro] é muito pouco entendeu? Do jeito que está tudo caro, não dá para nada, entendeu? Para quem toma remédio que nem no caso dela [...] (F4).

O médico disse que ela tem que tomar até o final da vida dela. É um remédio de alto custo que eu não tenho condições de comprar (F6).

Diante da indisponibilidade do medicamento na rede pública e da impossibilidade de comprálo, os familiares adotam algumas estratégias, entre elas, a encomenda de medicamento manipulado, a aquisição da medicação com outros pacientes e a reunião de membros da família para colaborar na compra.

Quando ela começou a tratar e o remédio, na época, ficava caro, aí mandamos manipular. Eu sei que o remédio não tava combatendo (F8).

No começo faltou, mas ela não chegou a ficar sem tomar não, porque tinha uma parente minha que tomava. Aí o médico trocou o remédio dela e ela deu os remédios para minha mãe. Aí ela não chegou a ficar sem (F8).

Aí eu consigo muitas vezes no $C$ [serviço de saúde]. Agora, se não tem [o medicamento], a gente fala: 'Vamos comprar né?'. Nós somos em quatro filhas. Aí, nós juntamos e compramos o remédio (F12).

\section{Identificando o uso de bebida alcoólica pelo paciente}

Outra dificuldade manifestada pelos familiares foi o consumo de bebidas alcoólicas pelo idoso com TAB. Seus depoimentos mostram que há pacientes que deixam de utilizar o medicamento quando ingerem bebida alcoólica. Destaca-se que não foram citadas pelos familiares estratégias adotadas diante desse problema.

Ele sai para beber um pouco e ele já fica bem alegre. De vez em quando ele traz uma cerveja. Eu falo: Você não vai tomar remédio? Ele diz que não, que vai tomar uma cerveja (F5).
Ela já fez [uso de álcool] quando ela está em crise, até hoje ela gosta de tomar uma cerveja (F12).

Ele toma a cervejinha dele até hoje (F13).

\section{DISCUSSÃO}

Com relação à caracterização dos sujeitos do estudo, destaca-se que apenas uma pequena parcela $(17,64 \%)$ dos mesmos possuía vínculo empregatício. A sobrecarga do familiar decorrente das funções relativas ao cuidado com o paciente pode ter sido um fator limitante para o trabalho com vínculo empregatício.

Mesmo sendo mencionados como as pessoas mais envolvidas no cuidado relacionado ao tratamento medicamentoso, provavelmente muitos familiares não estavam preparados para exercer tal função ou não a cumpriam efetivamente, pois a maioria dos mesmos não conhecia a dose e a frequência da medicação prescrita para o paciente. Este déficit de conhecimento pode ser prejudicial para o sucesso do tratamento desses pacientes. ${ }^{11}$ Destaca-se, ainda, que a falta de conhecimento dos familiares a esse respeito pode sinalizar uma falha na assistência oferecida pelos profissionais de saúde.

Destituídos do conhecimento sobre a dose e a frequência dos medicamentos utilizados pelos pacientes, os familiares podem ter limitações para intervir auxiliando-os a manter a medicação em níveis terapêuticos. Tal achado é agravado pelo fato de que muitos desses idosos necessitavam de auxílio para a administração de medicamentos. Dessa forma, podem se manter elevados os riscos de ineficácia do tratamento, pela utilização dos medicamentos abaixo dos níveis terapêuticos e o potencial risco de toxicidade para o idoso com $\mathrm{TAB}$, especialmente se considerarmos as particularidades dessa clientela na metabolização dos medicamentos.

São muitas as dificuldades vivenciadas pelos familiares relacionadas ao tratamento medicamentoso dos idosos com TAB. Entre tais dificuldades, identificou-se o julgamento ambíguo que os familiares fazem em relação aos efeitos do medicamento utilizado pelo paciente. A literatura revela que a avaliação dos efeitos da medicação de fato é conflituosa, especialmente porque o medicamento causa efeitos colaterais e diminui a probabilidade da ocorrência de crises e recaídas, mas não pode extingui-las definitivamente. ${ }^{16}$

A não aderência ao tratamento foi identificada pelos familiares como um problema relevante 
no tratamento dos idosos com TAB. Tal resultado é corroborado pela literatura, que aponta que aproximadamente $50 \%$ dos pacientes bipolares interrompem o tratamento pelo menos uma vez, enquanto $30 \%$ deles o fazem ao menos duas vezes ${ }^{17}$ e cerca de $1 / 3$ dos pacientes com TAB utilizam menos da metade da medicação prescrita. ${ }^{18}$

Os familiares assinalaram a não aderência como um comportamento que poderia ser intencional ou não intencional. A esse respeito, um estudo que investigou a aderência ao tratamento em adultos com TAB identificou que a maioria dos indivíduos não aderia à medicação por comportamento não intencional (paciente se esquece de tomar o medicamento ou é descuidado quanto ao horário do mesmo). ${ }^{19}$ Ressalta-se que, ao detectar a não aderência do paciente ao tratamento, os familiares adotavam estratégias para auxiliá-lo, demonstrando serem importantes parceiros do paciente e do profissional da saúde, na terapêutica medicamentosa.

No presente estudo, a não aderência foi considerada pelos familiares como causa e conseqüência de crises e recaídas. De fato, a literatura assinala que a não aderência ao tratamento medicamentoso em pacientes com TAB está associada a pior prognóstico, risco aumentado de recaídas e de suicídio. ${ }^{9}$

Foi possível verificar, por meio dos depoimentos, que as alterações na prescrição medicamentosa, em alguns momentos, geraram insegurança. Ressalta-se que pacientes e familiares devem ter espaço para expor seus temores e ser devidamente informados sobre os motivos das alterações na prescrição médica.

É válido ressaltar que os familiares identificaram o tratamento como inadequado em ocasiões diversas. A condução do tratamento de forma indevida pode ter sérias repercussões. É necessário atentar para a possibilidade de que o tratamento inadequado, oferecido em algum momento, possa gerar e perpetuar a insegurança dos familiares frente ao tratamento, e possa desmotivá-los na colaboração para a aderência ao tratamento do idoso com TAB.

Outra dificuldade relatada pelos sujeitos do estudo foi a preocupação com o acesso aos medicamentos. A garantia do acesso aos medicamentos na rede pública é de suma importância para favorecer a aderência ao tratamento. Este estudo verificou que muitas famílias tinham limitações financeiras para a compra dos medicamentos, especialmente daqueles considerados de "alto custo" pelo Ministério da Saúde. A esse respeito, estudo ${ }^{20}$ realizado em pacientes com condição crônica de saúde mostrou que $32,1 \%$ dos pacientes restringiram o uso do medicamento, também, devido ao custo. Esses pacientes apresentaram quase o dobro de chance de um declínio significativo na saúde em dois anos de seguimento. Desse modo, os profissionais de saúde devem conhecer o custo dos medicamentos prescritos para os pacientes e questioná-los regularmente sobre as possíveis dificuldades financeiras para continuidade da terapia medicamentosa.

Observou-se, também, nos depoimentos dos familiares o comprometimento do tratamento pela utilização de bebidas alcoólicas pelos idosos com TAB. Este é um problema relevante, visto que o uso indevido do álcool é a comorbidade mais associada ao TAB, sendo capaz de alterar a expressão, o curso e o prognóstico do transtorno. ${ }^{21}$ A literatura ${ }^{22}$ revela que em pacientes com TAB, o uso de álcool aumenta o risco de crises, principalmente as depressivas, tentativas de suicídio e internações, além de ser cerca de cinco vezes mais prevalente nos referidos pacientes do que na população geral.

Este estudo identificou que familiares de idosos com TAB possuem uma posição privilegiada para identificar problemas e intervir colaborando no tratamento. Entretanto, muitos deles encontram-se despreparados e sobrecarregados. No contexto da Reforma Psiquiátrica, que ora se apresenta, a família vem sendo resgatada para colaborar no diagnóstico, tratamento e manutenção do paciente na comunidade. Entretanto, é necessário que ela seja assessorada, preparada e apoiada pelos profissionais de saúde. ${ }^{23-25}$

A esse respeito, estudo ${ }^{26}$ identificou a necessidade de os integrantes da família terem suporte emocional para poder fortalecerem-se. Nesse aspecto, reside a importância de os profissionais estarem atentos às necessidades dos familiares a partir da realização de intervenções como grupo de familiares, visitas domiciliares, entre outras atividades que favoreçam a inserção dos mesmos no serviço, em uma perspectiva de que a família precisa ser cuidada, para que se sinta fortalecida e possa ter e oferecer suporte emocional.

\section{CONSIDERAÇÕES FINAIS}

Este estudo verificou o conhecimento e as dificuldades de 17 familiares de idosos com TAB, a respeito do tratamento medicamentoso, e identi- 
ficou baixo nível de conhecimento, especialmente sobre as doses e frequência dos medicamentos. São de suma importância as ações educativas junto aos familiares, pois a promoção de amplo conhecimento sobre os medicamentos utilizados pelos pacientes poderia contribuir para maior segurança do idoso com TAB na terapêutica medicamentosa. Ainda, ao confiar na proposta terapêutica, a família pode ajudar o paciente a aceitá-la e a encarar necessidade de tomar os medicamentos por longo tempo, garantindo a adesão ao tratamento.

Muitas dificuldades foram relatadas pelos familiares nos cuidados referentes ao tratamento medicamentoso dos idosos com TAB: ambigüidade em relação à avaliação dos benefícios do medicamento, não aderência dos pacientes ao medicamento, sobrecarga relacionada aos cuidados com os medicamentos, tratamento inadequado, preocupação com o acesso ao medicamento e utilização de bebida alcoólica pelo paciente. Constatase que a família é provedora de apoio e suporte de cuidado, quando uma enfermidade acomete o idoso com TAB. Nesta experiência, ela vivencia conflitos, dúvidas e sobrecarga em relação ao tratamento medicamentoso, provocando instabilidade permanente da vida cotidiana. Tais achados apontam para a necessidade de que os familiares sejam efetivamente incluídos no planejamento do cuidado ao paciente não só como parceiros, mas também como alvo das intervenções.

\section{REFERÊNCIAS}

1. Aziz R, Lorberg B, Tampi RP. Treatments for late-life bipolar disorder. Am J Geriatr Pharmacoter. 2006 Dez; 4(4):347-64.

2. Depp CA, Jeste DV. Bipolar disorder in older adults: a critical review. Bipolar Disord. 2004 Out; 6(5):343-67.

3. Almeida OP, Fenner S. Bipolar disorder: similarities and differences between patients with illness onset before and after 65 years of age. Int Psychogeriatr. 2002 Set; 14(3):311-22.

4. Benedetti A, Scarpellini P, Casamassima F, Lattanzi L, Liberti, M, Musetti L, et al. Bipolar disorder in late life: clinical characteristics in a sample of older adults admitted for manic episode. Clin Pract Epidemiol Ment Health. 2008 Jul; 29(4):22.

5. Zung SP. Estudo comparativo com ressonância magnética em idosos com transtorno afetivo bipolar usuários ou não de lítio [tese]. São Paulo (SP): Faculdade de Medicina da Universidade de São Paulo; 2007.

6. Gildengers AG, Mulsant BH, Begley A, Mazumdar S, Hyams AV, Reynolds CF, et al. The longitudinal course of cognition in older adults with bipolar disorder. Bipolar Disord. 2009 Nov; 11(7):744-52.

7. Scheinkman L. Transtorno bipolar no idoso: estado atual do conhecimento. Arq Bras Psiquiatr Neurol Med Legal. 2006 Jan-Mar; 100(1):34-7.

8. Miasso AI, Cassiani SHB, Pedrão LJ. Transtorno afetivo bipolar e terapêutica medicamentosa: identificando barreiras. Rev Latino-am Enfermagem. 2008 Jul-Ago; 16(4):739-45.

9. Pompili M, Serafini G, Del Casale A, Rigucci S, Innamorati M, Girardi P, et al. Improving adherence in mood disorders: the struggle against relapse, recurrence and suicide risk. Expert Rev Neurother. 2009 Jul; 9(7):985-1004.

10. Colvero LA, Costardi Ide CA, Rolim MA. Família e doença mental: a difícil convivência com a diferença. Rev Esc Enferm USP. 2004 Jun; 38(2):197-205.

11. Souza RS, Scatena MCM. Produção de sentidos acerca da família que convive com o doente mental. Rev Latino-am Enfermagem. 2009 Mar-Abr; 13(2):173-9.

12. Miklowitz DJ. The role of the family in the course and treatment of bipolar disorder. Curr Dir Psychol Sci. 2007 Ago; 16(4):192-6.

13. Stape DDB. O conhecimento do paciente com alta hospitalar sobre a continuidade do seu tratamento [dissertação]. São Paulo (SP): Escola de Enfermagem da Universidade de São Paulo; 1979.

14. Miasso, AI. Terapêutica medicamentosa: orientação e conhecimento do paciente na alta e pós-alta hospitalar [dissertação]. Ribeirão Preto (SP): Escola de Enfermagem de Ribeirão Preto da Universidade de São Paulo; 2002.

15. Minayo MCS. O desafio do conhecimento: pesquisa qualitativa em saúde, 6a ed. São Paulo (SP): Hucitec; 1999.

16. Miasso AI. "Entre a cruz e a espada": o significado da terapêutica medicamentosa para a pessoa com transtorno afetivo bipolar, em sua perspectiva e na de seu familiar [tese]. Ribeirão Preto (SP): Escola de Enfermagem de Ribeirão Preto da Universidade de São Paulo; 2006.

17. Lingam R, Scott J. Treatment non-adherence in affective disorders. Acta Psychiatr Scand. 2002 Mar; 105; 164-72.

18. Sajatovic M, Davies M, Hrouda DR. Enhancement of treatment adherence among patients with bipolar disorder. Psychiatr Serv. 2004 Mar; 55(3):264-9.

19. Miasso AI, Monteschi M, Giacchero KG. Transtorno afetivo bipolar: adesão ao medicamento e satisfação com o tratamento e orientações da equipe de saúde de um núcleo de saúde mental. Rev Latino-am Enfermagem. 2009 Jul-Ago; 17(4):548-56.

20. Heisler M, Langa KM, Eby EL, Fendrick M, Kabeto UM, Piette JD. The health effects of restricting prescription medication use because of cost. Medical Care. 2004 Jul; 42(7):626-34. 
21. Levin FR, Hennesy G. Bipolar disorder and substance abuse. Biol Psychiatry. 2004 Nov; 56(10):738-48.

22. Kessler RC. The epidemiology of dual diagnosis. Impact of substance abuse on the diagnosis, course, and treatment of Mood Disorders. Biol Psychiatry. 2004 Nov; 56(10):738-48.

23. Barroso SM, Bandeira M, Nascimento E. Sobrecarga de familiares de pacientes psiquiátricos atendidos na rede pública. Rev Psiquiatr Clín. 2007; 34(6):270-7.

24. Navarini V, Hirdes A. A família do portador de transtorno mental: identificando recursos adaptativos. Texto Contexto Enferm. 2008 Dez; 17(4):680-8.

25. Macêdo VCD, Monteiro ARM. Enfermagem e a promoção de saúde mental na família: uma reflexão teórica. Texto Contexto Enferm. 2004 Out- Dez; 13(4):585-92.

26. Bielemann VLM, Kantorski LP, Borges LR, Chiavagatti FG, Willrich JQ, Souza AS, Heck R M. A inserção da família nos centros de atenção psicossocial sob a ótica de seus atores sociais. Texto Contexto Enfermagem. 2009 Jan-Mar; 18(1):131-9. 